\title{
Update on the COVID-19 Vaccine Research Trends: A Bibliometric Analysis
}

\author{
ZhaoHui Xu ${ }^{1,2}$ \\ Hui Qu ${ }^{1,2}$ \\ YanYing Ren' \\ ZeZhong Gong ${ }^{1,2}$ \\ Hyok Ju Ri (iD ${ }^{1-3}$ \\ Fan Zhang' \\ XiaoLiang Chen' \\ Wanji Zhu' \\ Shuai Shao' \\ Xin Chen (D)
}

'Department of Hernia and Colorectal Surgery, The Second Hospital of Dalian Medical University, Dalian, II6023, People's Republic of China; ${ }^{2}$ Dalian Medical University, Dalian, People's Republic of China; ${ }^{3}$ Department of Colorectal Surgery, The Hospital of Pyongyang Medical College, Pyongyang, 999093, People's Republic of Korea
Background: The coronavirus disease 2019 (COVID-19) pandemic is ravaging the world. To date, there are no standard therapies available to cure the disease. Consequently, research on COVID-19 vaccines is booming. This report aimed to assess the research trends of the global COVID-19 vaccines.

Methods: The relevant publications on the COVID-19 vaccines were searched in the Web of Science Core Collection Database (WOSCC) database from December 2019 to 11 August 2021. The VOSviewer1.6.16 was used to assess the co-authorship, cooccurrence, citation of countries, institutions, authors, journals, and hotspot keywords. The HistCite $^{\mathrm{TM}}$ (http://www.histcite.com/) software was used to calculate the total local citation score (TLCS) and total global citation score (TGCS) of each variable and generate the citation historiography graph of COVID-19 vaccine development using the citation time series analysis method.

Results: A total of 5070 studies authored by 21,151 researchers and published by 1364 different journals were eventually included in this study. The bulk of the retrieved studies were original articles $(n=2401,47.36 \%)$. Among these studies, $1204(23.75 \%)$ were published in 2020. A total of 3863 (76.19\%) were published in 2021 and $4295(84.71 \%)$ were open access. The highest number of studies was conducted in the USA, followed by England, China, and Germany. The main partners of the USA were China, England, and Canada. The University of Maryland (TLCS: 1618, TGCS: 2991) and Prof. Ugur Sahin from the University Medical Center of the Johannes Gutenberg University (TLCS: 1397, TGCS: 2407) were the most cited institution and author, respectively. The vaccines featured the highest number of papers, with 294 publications (TLCS: 0, TGCS: 1226). The most cited journal was the New England Journal of Medicine (TLCS: 3310, TGCS: 5914), with an impact factor (IF) of 91.245. The related topics included the following six aspects: attitudes towards vaccination, immunoinformatics analysis, clinical research, effectiveness and side effects, and the public management of vaccines. The timing diagram revealed that the research hotspots focused on the side effects of vaccines and public attitude towards vaccination.

Conclusion: This novel comprehensive bibliometric analysis can help researchers and nonresearchers to rapidly identify the potential partners, landmark studies, and research topics within their domains of interest. Through this study, we hope to provide more data to combat the COVID-19 pandemic.

Keywords: bibliometric analysis, COVID-19, vaccines

\section{Introduction}

The World Health Organization (WHO) declared the coronavirus disease 2019 (COVID-19) a pandemic on 11 March 2020. ${ }^{1}$ The most common manifestations of
Correspondence: Xin Chen

Tel +86 I 7709872266

Email chenxincjz@gmail.com 
COVID-19 are cough, fever, and fatigue, often accompanied by lung infection. As of 6:00 pm CEST on 19 August 2021, a total of 209,201,939 patients with COVID-19 were identified in more than 200 countries, areas, or territories, including 4,390,467 deaths, ${ }^{2}$ which further revealed that this substantial global impact of the pandemic (Figure 1). Currently, patients with COVID-19 are treated using antiviral drugs, antimalarial drugs, cell- and plasma-based therapy, traditional Chinese medicine, and monoclonal antibody therapy. ${ }^{3-5}$ Unfortunately, there are no standard therapies to cure the disease. However, a significant proportion of the population in several countries has not yet been infected and remains non-immune to severe acute respiratory syndrome coronavirus 2 (SARS-CoV-2). ${ }^{6}$ Therefore, vaccination remains the most economical and effective approach to prevent viral infection, especially in undeveloped countries. Vaccines are being developed on an unprecedented scale at a remarkable speed. To date, several vaccines have been approved for comprehensive marketing and use in the population.

Bibliometric analysis is based on the unique parameters of publications (such as countries, institutions, and author), which uses a combination of mathematics and statistical methods to quantitatively describe the current status, research hotspots, and trends in science and technology. ${ }^{7}$ Researchers and non-researchers can use the information to achieve a special purpose within their domains of interest (for example, identify active partners, landmark studies, research themes, and other useful information). Currently, COVID-19 vaccine-related research is booming. Researchers need to gain relevant knowledge regarding the different vaccines, however scattered information makes this process timeconsuming and laborious. We aimed to solve these problems by providing more data to combat the COVID-19 pandemic.

\section{Methods}

We searched the Web of Science Core Collection (WOSCC) database from December, 2019 to August 11, 2021, using a combination of relevant Medical Subject Heading terms and keywords: (vaccin* OR autovaccine* OR autogenous vaccine* OR active immunization* OR mass immunization* OR mass vaccination*) AND (COVID-19 OR COVID 19 OR COVID-19 virus disease* OR COVID 19 virus disease OR COVID-19 virus infection* OR COVID 19 virus infection OR
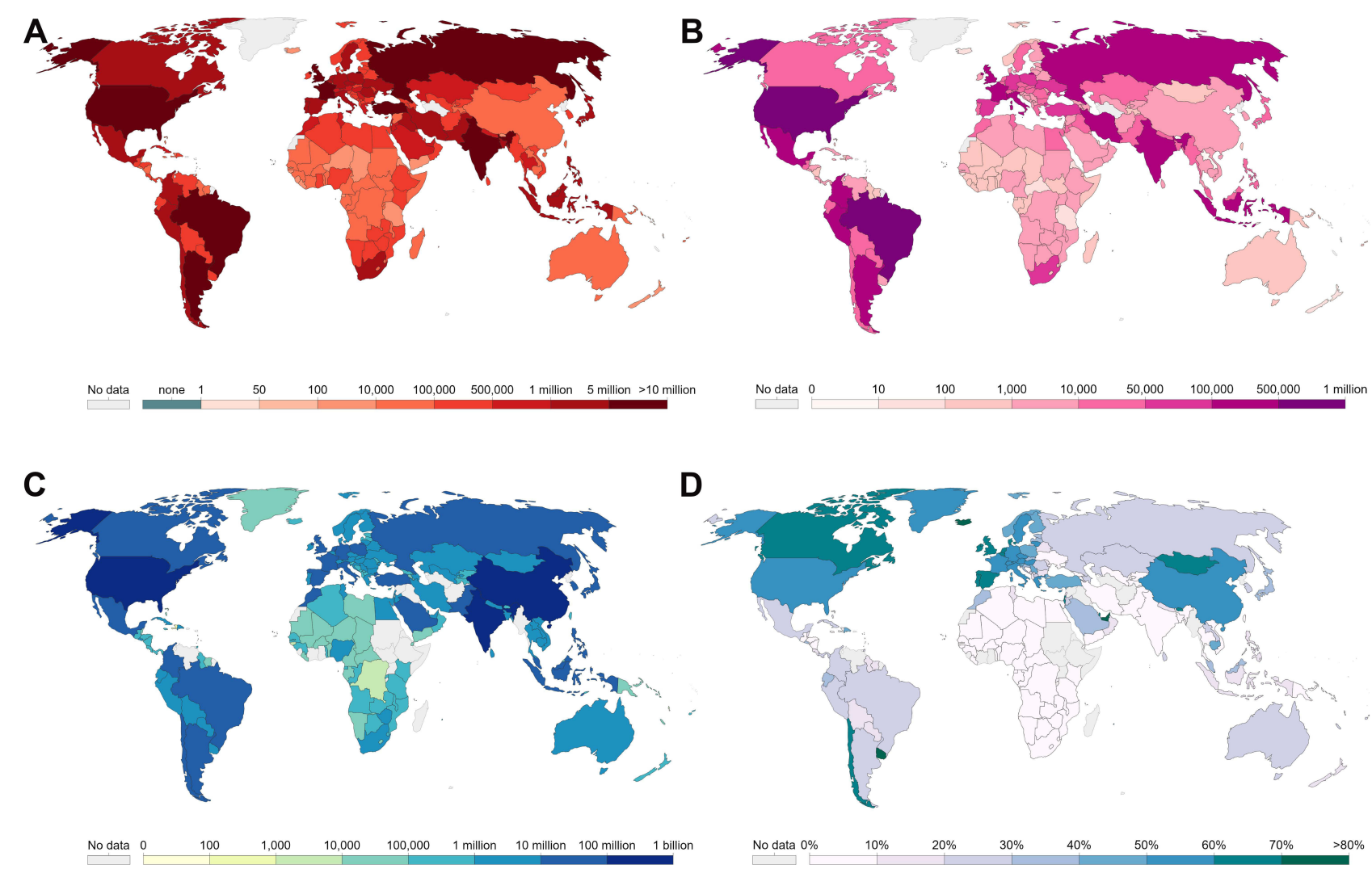

Figure I The statistics on the COVID-19 pandemic for every country in the world from Our World in Data. (A) The cumulative confirmed COVID-I9 cases in the world. (B) The cumulative confirmed COVID-19 deaths in the world. (C) The number of fully vaccinated individuals against COVID-19 (total number of people who have received all doses based on vaccination protocol). (D) Share of the fully vaccinated population against COVID-19. 
2019-nCoV infection* OR 2019 nCoV infection OR coronavirus disease-19 OR coronavirus disease 19 OR 2019 novel coronavirus disease OR 2019 novel coronavirus infection OR 2019-nCoV disease* OR 2019 nCoV disease OR coronavirus disease 2019 OR SARS coronavirus 2 infection OR SARSCoV-2 infection* OR SARS CoV 2 infection OR COVID-19 pandemic* OR COVID 19 pandemic OR severe acute respiratory syndrome coronavirus 2 OR new coronavirus).

Two investigators independently screened the literature, collected and crosschecked the information. A third author resolved the disagreements. Excel 2010 was used to manage the extracted data and perform statistical analysis. VOSviewer1.6.16 was used to access the co-authorship, co-occurrence, and citation of countries, institutions, authors, hotspot keywords, and journals. The size of the node represents the occurrence frequency of the relevant parameters, the thickness of the connection between nodes represents the degree of association, and different colors represent the different modules in the visualization mapping. The HistCite ${ }^{\mathrm{TM}}$ (http://www.histcite.com/) software was used to calculate the total local citation score (TLCS) and total global citation score (TGCS) of each variable and generate the citation historiography graph of COVID-19 vaccine development using the citation time series analysis method. TLCS refers to the citation frequency of a study in the software, and TGCS refers to the citation frequency in the WOSCC database. The current impact factor (IF) of the journals was obtained from the Journal Citation Reports of Thomson Reuters on June 30, 2021.

\section{Results}

\section{Publication Output}

A total of 9920 publications were identified from the WOSCC database. After independent screening by the two investigators, 5070 relevant publications were selected. These included $2401(47.36 \%)$ original research articles, $640(12.62 \%)$ review articles, 976 (19.25\%) editorial materials, $611(12.05 \%)$ early access manuscripts, 599 (11.81\%) letters, and 454 (8.95\%) other articles including meeting abstract, among other publications (Figure 2). Of these, 1204 (23.75\%) papers were published in 2020 and $3863(76.19 \%)$ were produced in 2021 (Figure 2). A total of 4295 (84.71\%) papers were open access.

\section{Co-Authorship Countries, Institutions, and Authors}

The top ten countries, institutions, and authors with the most publications are listed in Table 1. There were 69 countries with more than eight publications. The highest number of studies was conducted in the USA, followed by England, China, and Germany. Six clusters were obtained based on this information (Figure 3). Among them, the USA had 66 partners, and the total link strength (TLS) was 610 with 1681 publications. The primary collaborators of the USA were China, England, and Canada.

It was observed that 6321 institutions published the aforementioned studies and 176 of them published more than 12 studies. The clustered heatmap of the institutions is shown in Figure 3. The University of Oxford has published 111 related papers with 2219 global citations. The main collaborators of the university were the London School of Hygiene and Tropical Medicine and Public Health England. Six clusters were obtained from the analysis. The red zone (Cluster1, 67 items) represented the American institutions, such as Harvard Medical School (links: 74, TLS: 189, publications: 100), University of Washington, Standford University, Johns Hopkins University, University of Pennsylvania, Emory University. Cluster 2 comprised 41 items and
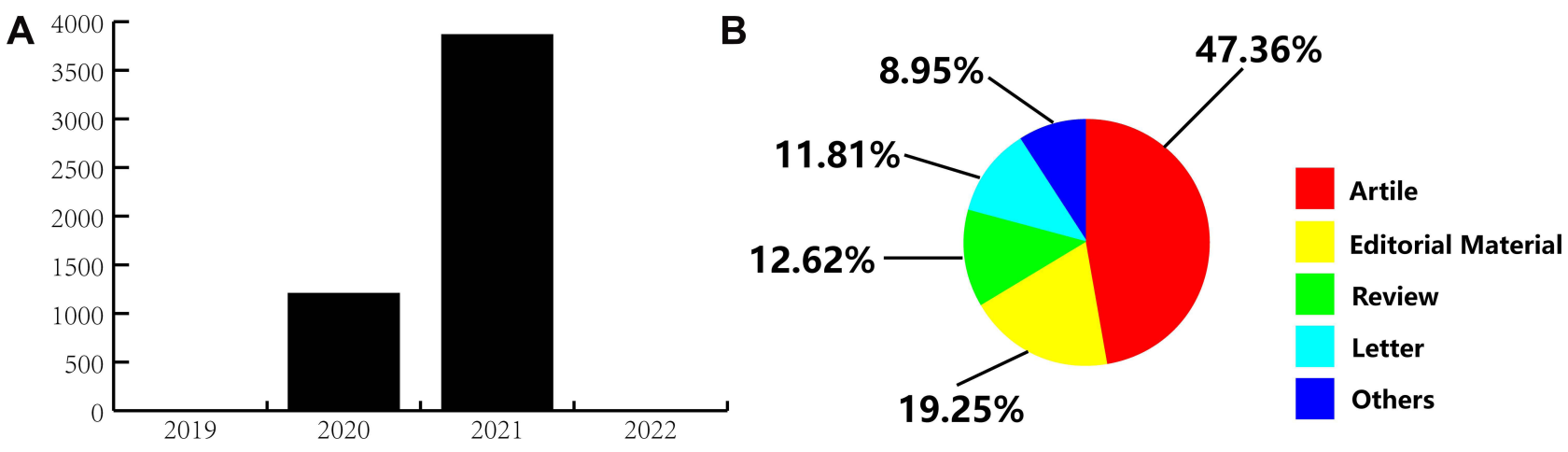

Figure 2 Bibliometric analysis of the COVID-19 publication. (A) Time trends summary. (B) Study type composition summary. 
Table I The Top Ten Most Active Countries, Organizations and Authors

\begin{tabular}{|c|c|c|c|c|c|}
\hline Subject & Records & Percentage (\%) & TLCS & TGCS & TLS \\
\hline \multicolumn{6}{|l|}{ Countries } \\
\hline USA & 1681 & 33.16 & 8567 & $20,48 \mid$ & 21,715 \\
\hline UK & 565 & 11.14 & 3678 & 7772 & 10,975 \\
\hline Italy & 388 & 7.65 & 525 & 1594 & 5066 \\
\hline Peoples R China & 383 & 7.55 & 1747 & 5560 & 7681 \\
\hline India & 319 & 6.29 & 276 & 1723 & 3668 \\
\hline Germany & 299 & 5.90 & 2203 & 4555 & 6753 \\
\hline Canada & 249 & 4.91 & 667 & 1631 & 3677 \\
\hline Australia & 179 & 3.53 & 531 & 1289 & 2874 \\
\hline France & 177 & 3.49 & 278 & 723 & 2437 \\
\hline Spain & 167 & 3.29 & 430 & 734 & 2343 \\
\hline \multicolumn{6}{|l|}{ Organizations } \\
\hline Univ Oxford & 111 & 2.19 & 1384 & 2880 & 2032 \\
\hline Harvard Med Sch & 100 & 1.97 & 710 & 1433 & 1776 \\
\hline Univ Washington & 80 & 1.58 & 81 & 532 & 1437 \\
\hline London Sch Hyg \& Trop Med & 76 & 1.50 & 77 & 897 & 1096 \\
\hline Johns Hopkins Univ & 60 & 1.18 & 114 & 522 & 688 \\
\hline Univ Penn & 60 & 1.18 & $15 \mid$ & 414 & 794 \\
\hline Tel Aviv Univ & 59 & 1.16 & 268 & 487 & 726 \\
\hline Univ Toronto & 58 & 1.14 & 174 & 261 & 544 \\
\hline Imperial Coll London & 57 & 1.12 & 427 & 971 & 1052 \\
\hline Emory Univ & 56 & 1.10 & 1139 & 2028 & 1794 \\
\hline \multicolumn{6}{|l|}{ Authors } \\
\hline Elisabeth Mahase & 57 & 1.12 & 0 & 253 & 49 \\
\hline Gareth lacobucci & 25 & 0.49 & 0 & 33 & 3 \\
\hline Kuldeep Dhama & 21 & 0.41 & 72 & 301 & 157 \\
\hline Owen Dyer & 17 & 0.34 & 0 & 26 & 4 \\
\hline Lindsey Robert Baden & 16 & 0.32 & 520 & 834 & 0 \\
\hline Peter Jay Hotez & 16 & 0.32 & 195 & 492 & 148 \\
\hline Janice Hopkins Tanne & 15 & 0.30 & 0 & 22 & 8 \\
\hline Jacqui Wise & 15 & 0.30 & 0 & 60 & 11 \\
\hline Maria Elena Bottazzi & 14 & 0.28 & 203 & 460 & 139 \\
\hline Eric J. Rubin & 14 & 0.28 & 9 & 17 & I \\
\hline
\end{tabular}

represented the European academic institutions dominated by the UK, namely the University of Oxford, London School of Hygiene and Tropical Medicine and the University of Cambridge. Cluster 3 comprised 24 items and represented academic institutions dominated by Toronto, such as the University of Toronto, McGill University, and McMaster University. The orange zone (Cluster 4, 15 items) represented Chinese institutions, such as Fudan University, Chinese Academy of Sciences, and The University of Hong Kong. Cluster 5 (shown in purple) comprised 15 items and represented European academic institutions, namely the University of Paris, Aix-Marseille University, Sorbonne University, University of Bern, and University of Munich, Germany.
Cluster 6 (shown in bright blue) comprised 14 items and represented academic institutions in Italy, such as the University of Padua, University of Milan, University of Genoa, and University of Florence.

A total of 21,151 authors have published papers on COVID-19 vaccines. The relevant clustered heatmap is shown in Figure 3. Eight clusters were obtained from the analysis. Among the authors, Prof. Mahase Elisabeth has published 57 papers with 254 global citations.

\section{Author Keywords}

We manually standardized the keywords before constructing the cluster map. We merged and replaced similar keywords, such as adverse events, clinical trial, and vaccination 

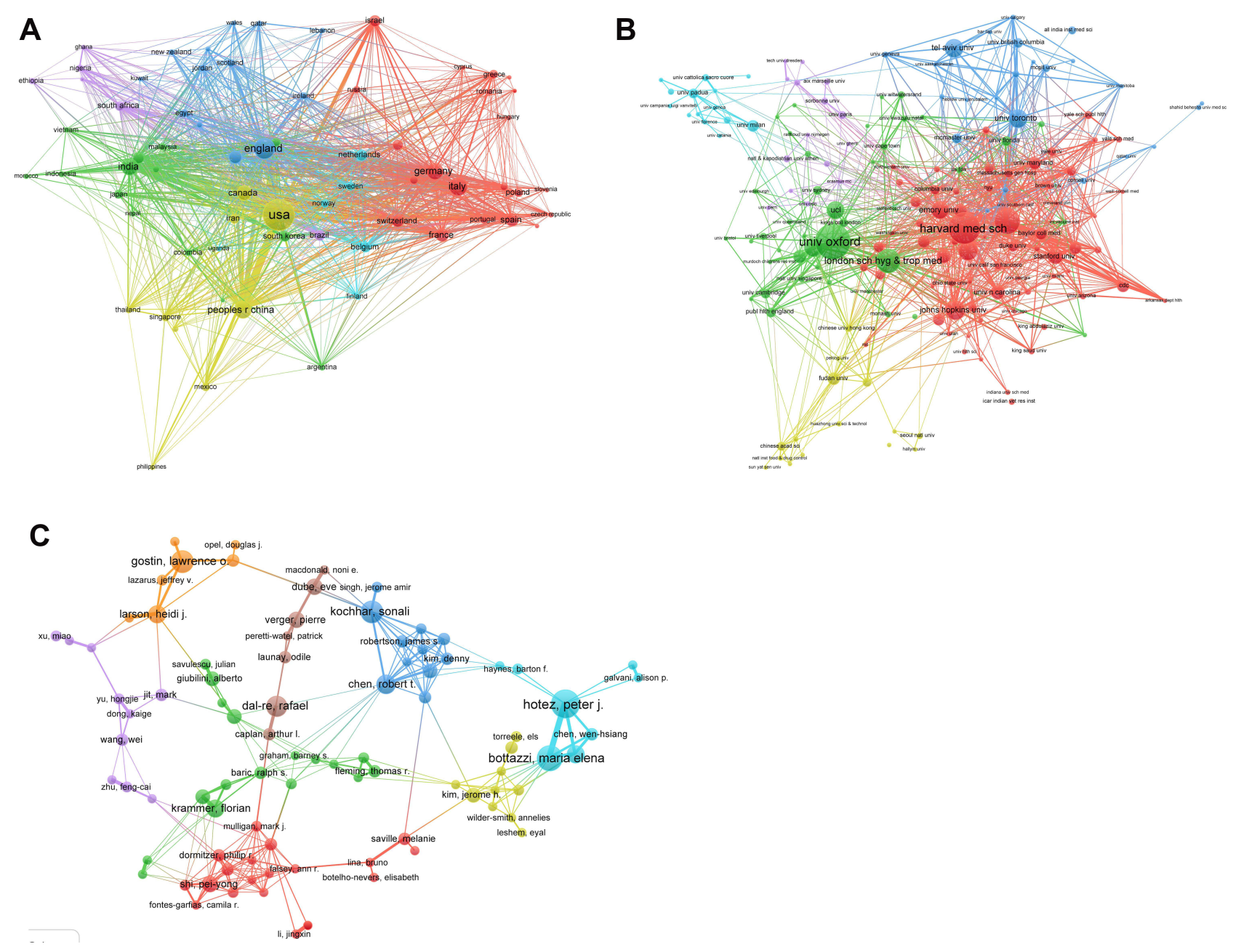

Figure 3 Bibliometric analysis of co-authorship. (A) Country. (B) Institution. (C) Author.

hesitancy with adverse effects, clinical trials, and vaccine hesitancy, respectively. A total of 129 keywords were considered in the analysis (occurrence $>8$ ). The keyword that appeared commonly was "SARS-CoV-2" (links: 128, occurrences: 2080, TLS: 1885) that demonstrated a strong association with "vaccine", "vaccination", "vaccine hesitancy", and "coronavirus". Six clusters were obtained from the analysis (Figure 4). Cluster 1 comprised 43 items that represented the attitudes to vaccination (shown in red) and included keywords such as vaccination hesitancy, acceptance, vaccination confidence, intention, attitudes, willingness, trust, risk perception, herd immunity, social media and misinformation. Cluster 2 comprised 26 items that represented the immunoinformatics on vaccines (shown in green) and included keywords such as spike, spike protein, subunit vaccine, vaccine design, vaccine development, epitope, DNA vaccine. Cluster 3 comprised 18 items that represented the clinical research on the vaccines (shown in blue) and included keywords such as clinical trials, bioinformatics, prevention, BCG, drug, and mathematical model. Cluster 4 comprised 17 items that represented the vaccine effectiveness (shown in yellow) and included keywords such as safety, efficacy, immune response, older adult, surveillance, vaccination. Cluster 5 includes 14 items and represents the side effects of vaccines (shown in purple), including keywords such as adverse effects, anaphylaxis, hypersensitivity, immunization, antibody response, polyethylene glycol, lipid nanoparticles, and Pfizer. Cluster 6 comprised 11 items that represented the public management (shown in bright blue) and included keywords such as public health, global health, health policy, ethic, and children. The timing diagram showed that the research hotspots focused on the side effects of the vaccines and public attitudes towards vaccination. 


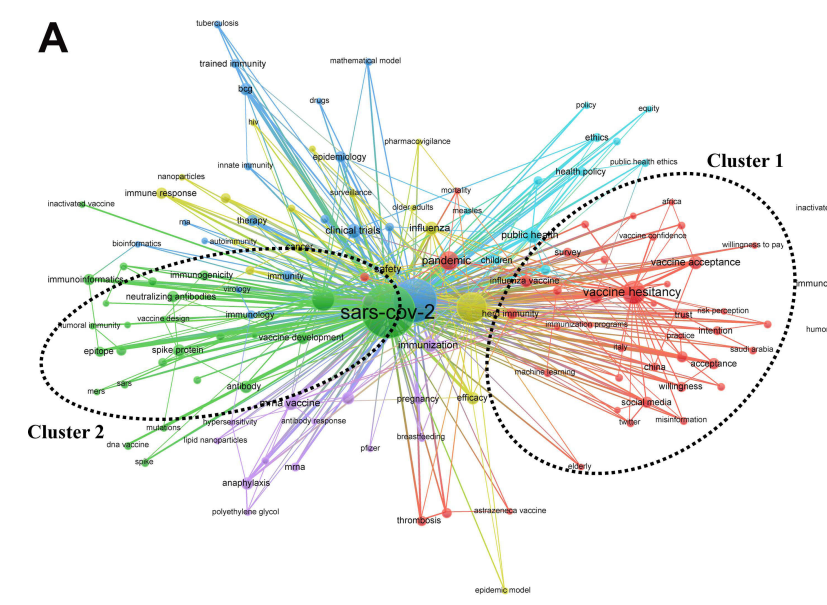

Figure 4 Bibliometric analysis of keywords. (A) Cluster analysis. (B) Timing analysis.

\section{Citations and Publications}

Compared with the number of publications, the frequency of citations of each study may better reflect the influence and importance. Moreover, TLCS is more objective compared with TGCS. Twelve papers were authored by Prof. Ugur Sahin from the University Medical Center of the Johannes Gutenberg University (TLCS: 1397, TGCS: 2407) and 39 papers were from the University of Maryland (TLCS: 1618, TGCS: 2991); therefore, the two were the most cited author and institution, respectively. According to the WOSCC database, COVID-19 vaccines from the USA were cited most frequently ( $\mathrm{n}=1678$, TLCS: 8567, TGCS: 20,481) (Figure 5).

A total of 1364 journals published the relevant papers; of which, 133 published more than seven papers. In total, 1291 papers were published in the top ten active journals that accounted for $25.5 \%$ of the publications in the WOSCC database. The Vaccines featured the maximum number of papers, with 294 publications (TLCS: 0, TGCS: 1226). The most cited journal was the New England Journal of Medicine (TLCS: 3310, TGCS: 5914), with an IF of 91.245 (Figure 5).

The top 100 most cited studies on COVID-19 vaccines are listed in Table S1. A majority of the studies were clinical or preclinical trials regarding vaccine safety, efficacy and immunogenicity, whereas others were observational studies focused on the attitudes towards vaccination. The mean TLCS of the top 100 most cited studies was 97.88 (range, 30-827). In 2020, 83 papers were published; of which, 42 were authored by American researchers. The Lancet (14 papers with 3229 citations) and the New
England Journal of Medicine (14 papers with 5297 citations) published the maximum number of papers.

A citation historiography graph based on the studies with the top 100 TLCS is shown in Figure 6. Furthermore, we identified some landmark studies, namely ID1686, ${ }^{8}$ ID $991,{ }^{9}$ ID2037, ${ }^{10}$ and ID2308 $^{11}$ (Figure 6 and Table S1). Considering the rapid outbreak of the COVID-19 pandemic, several research topics have emerged simultaneously in large numbers in a short period, becoming research frontiers.

\section{Discussion}

COVID-19 has spread globally, and there is an ongoing battle against the disease. We are all in the midst of the epidemic. Considering the novelty, there are several familiar and unfamiliar aspects of the disease and the virus. Our pre-search of the WOSCC database revealed that at least 110,000 studies have been published on COVID-19. Several vaccines have been approved for full marketing and use. With the ongoing resurgence of COVID-19 and the continuous mutation of the virus globally, there is an urgent need for safe and effective novel vaccines. As of 17 August 2021, 32.2\% of the global population has received at least one dose of a COVID-19 vaccine, and $24.2 \%$ are fully vaccinated (Figure 1). ${ }^{12}$ However, only $1.3 \%$ of the population in lower-income countries has received at least one dose of a vaccine. Therefore, vaccination programs remain important.

Global efforts have promoted the research of vaccine. Unlike some previous bibliometric studies on COVID-19 related topics, ${ }^{13,14}$ we have expanded on the latest data and focused solely on vaccines. Such an analysis is crucial 
A

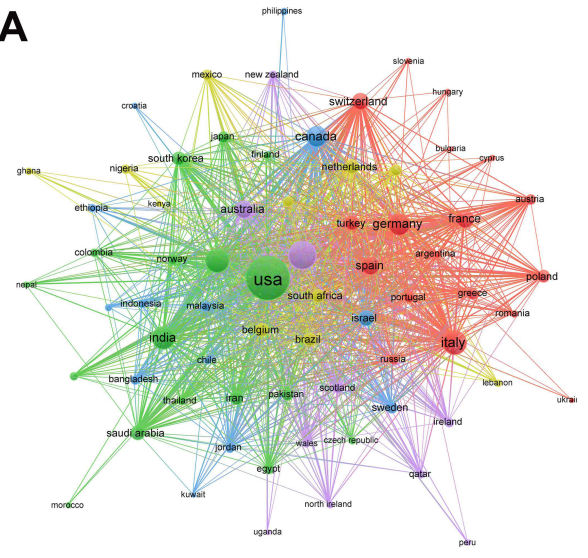

C

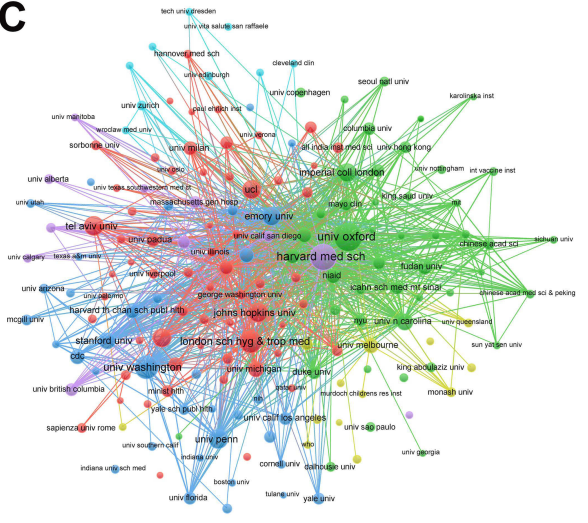

E

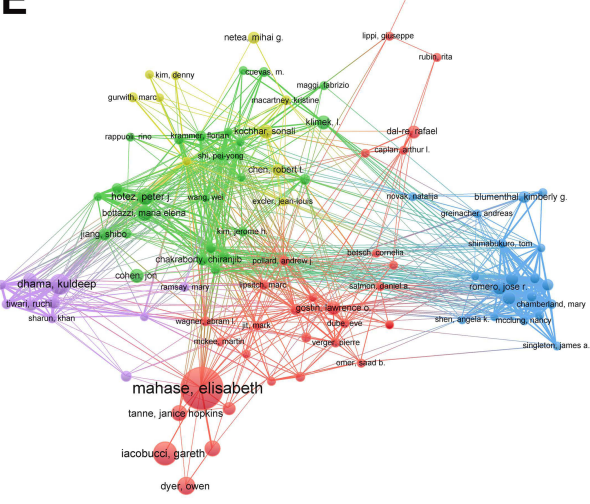

G

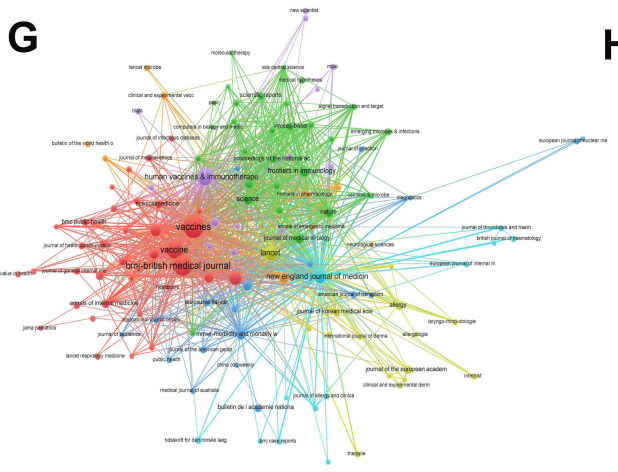

B

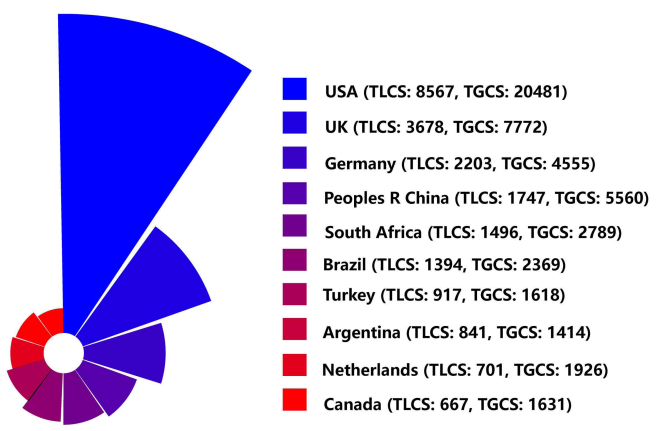

$\mathbf{F}$

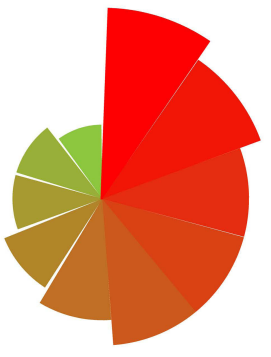

Univ Maryland (TLCS: 1618, TGCS: 2991)

NIAID (TLCS: 1521, TGCS: 3240)

Vanderbilt Univ (TLCS: 1401, TGCS: 2379)

BioNTech (TLCS: 1396, TGCS: 2403)

Univ Oxford (TLCS: 1384, TGCS: 2880)

Pfizer (TLCS: 1247, TGCS: 2190)

Clicinnati Childrens Hosp (TLCS: 1219, TGCS: 2064)

Emory Univ (TLCS: 1139, TGCS: 2028)

Modema (TLCS: 1139, TGCS: 2035)

Johns Hopkins Bloomberg Sch Publ HIth (TLCS: 952, TGCS: 1751)
$\mathbf{F}$

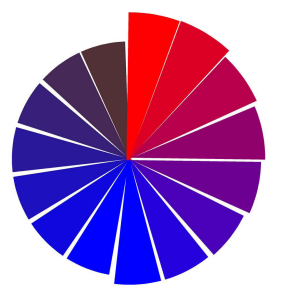

H

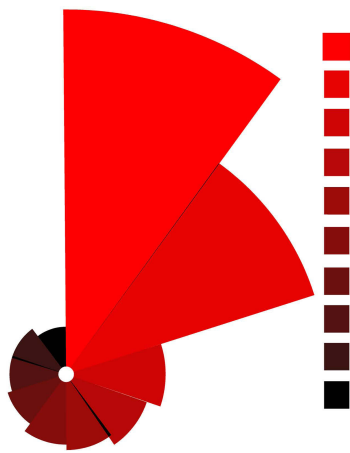

Sahin U (TLCS: 1397, TGCS: 2407)

Tureci O (TLCS: 1397, TGCS: 2407)

Dormitzer PR (TLCS: 1396, TGCS: 2472)

Swanson KA (TLCS: 1394, TGCS: 2467)

Jansen KU (TLCS: 1359, TGCS: 2326)

Graham BS (TLCS: 1351, TGCS: 2406)

Cooper D (TLCS: 1343, TGCS: 2361)

Li P (TLCS: 1231, TGCS: 2072)

Absalon J (TLCS: 1219, TGCS: 2063)

Bailey R (TLCS: 1219, TGCS: 2063)

Gruber WC (TLCS: 1219, TGCS: 2063)

Gurtman A (TLCS: 1219, TGCS: 2063)

Kitchin N (TLCS: 1219, TGCS: 2063)

Koury K (TLCS: 1219, TGCS: 2063)

Lockhart S (TLCS: 1219, TGCS: 2063)

NEW ENGLAND JOURNAL OF MEDICINE (TLCS: 3310, TGCS: 5914)

LANCET (TLCS: 2271, TGCS: 4008)

NATURE (TLCS: 830, TGCS: 1926)

SCIENCE (TLCS: 732, TGCS: 1735)

VACCINE (TLCS: 694, TGCS: 946)

JAMA (TLCS: 555, TGCS: 982)

MMWR (TLCS: 521, TGCS: 859)

LANCET INFECTIOUS DISEASES (TLCS: 396, TGCS:698)

NATURE MEDICINE (TLCS: 378, TGCS: 638)

HUMAN VACCINE \& IMMUNOTHERAPEUTICS (TLCS: 337, TGCS: 677)

Figure 5 Bibliometric analysis of citations. (A and B) Countries. (C and D) Organizations. (E and F) Authors. (G and H) Journals. (B, D, F, and H) The top I0 most cited countries, institutions, authors, and journals, respectively. 

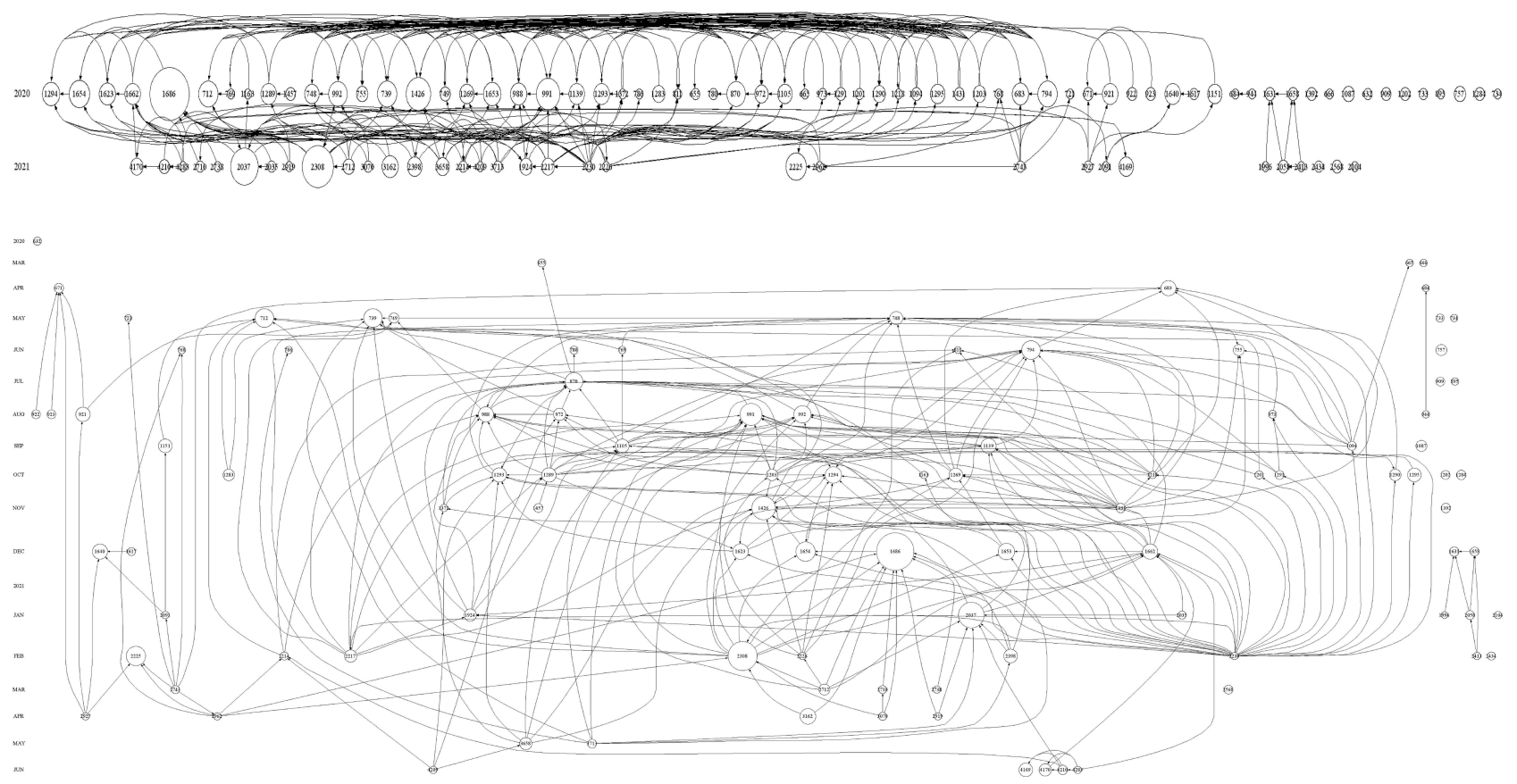

Figure 6 The citation historiography graph based on the studies with the top 100 TLCS. Abbreviation: TLCS, total local citation score.

considering the large number of literature and rapidly evolving research on COVID-19. To the best of our knowledge, this is the most recent and comprehensive bibliometric analysis on the subject. The 5070 publications on the COVID-19 vaccines indexed in the WOSCC database were analyzed in the current study. Among these, 1204 papers were published in 2020 and 3863 were published in 2021. We predict that relevant research will continue until the pandemic is controlled. The USA, the UK, Italy, and China have made outstanding contributions to this important field. The University of Oxford and the University of Maryland are the most active institutions. The high-impact medical journals such as the New England Journal of Medicine, Lancet, Nature, Science, Cell, Immunity, Allergy, and Journal of the American Medical Association published the COVID-19 vaccinerelated studies, which defined the direction of research. ${ }^{15}$ Furthermore, $84.75 \%$ of the papers were open access, which significantly advanced the related research. Ugur Sahin was the most cited author with 1397 peer citations, and his most cited study $(827 \text { times })^{8}$ was published in 2020. His research focused on two lipid nanoparticleformulated and nucleoside-modified RNA vaccine candidates namely BNT162b1 and BNT162b2. The results of the associated clinical trials revealed the effectiveness and safety of vaccines.
As the most frequently used keyword, "SARS-CoV-2" was strongly associated with "vaccine", "vaccination", "vaccine hesitancy", and "coronavirus". The COVID-19 vaccine research hotspots were as follows: attitudes towards vaccination, immunoinformatics analysis, clinical research, the effectiveness and side effects, and the public management of vaccines. The timing diagram showed that the research hotspots focused on the side effects of vaccines and public attitudes towards vaccination. The largest cluster represented the attitudes of the population towards vaccination. Many adults are willing to take a COVID-19 vaccine; however, a significant number of individuals were vaccine-hesitant. Vaccine hesitancy, defined as patientlevel reluctance to get vaccinated, has been considered one of the top ten global health threats of 2019. ${ }^{16}$ Misinformation and unsubstantiated rumors regarding the benefits, vaccine components, and side effects of vaccination limits patient understanding and complete acceptance. ${ }^{17}$ In addition, it must be noted that social media may erroneously propagate anti-vaccine campaigns and fuel vaccine hesitancy. ${ }^{18}$ Several factors have been reported to influence the response to vaccination, such as sex, psychological health, race, educational level, influenza vaccination history and trust in the government. ${ }^{17,19,20}$ Therefore, a targeted multi-pronged effort is required to maximize vaccination and coverage. 
Clusters 2 and 3 comprising 44 items focused on immunological and clinical research and included keywords such as spike protein, subunit vaccine, vaccine design, vaccine development, epitope, clinical trials, bioinformatics, BCG, and drug. The spike protein is an essential component that mediates virus invasion and is considered as a target to develop potential vaccines and antiviral drugs. Currently, there are some successful SARS-CoV-2 spike-based vaccines, such as BNT162b2 (PfizerBioNTech), mRNA-1273 (Moderna), ChAdOx1 nCov-19 (AstraZeneca), and Ad26.COV2.S (Johnson \& Johnson/ Janssen). However, recent studies have reported that some SARS-CoV-2 spike-based vaccines might elicit protective antibodies capable of preventing the production of GM-CSF and the progression of COVID-19. ${ }^{21}$ In contrast to the conventional approach, some researchers have attempted to design a multi-epitope or peptide-based COVID-19 vaccine with the aid of immunoinformatics tools. $^{22,23}$ Until such new vaccines are developed, booster BCG vaccination may be beneficial to prevent COVID-19 infection. $^{24}$ As of August 20, 2021, there are 296 vaccine candidates under development, of which 112 are in the clinical development stage. $^{25}$ The vaccine platforms include protein subunit, viral vector (replicating or nonreplicating), DNA, RNA and inactivated virus. The battle for vaccine development will continue in the challenge.

The novel research hotspot in cluster 5 was adverse events after vaccination. The most common adverse effects include injection-site reactions, headache, fatigue, pyrexia, and chills. ${ }^{26}$ The rates of anaphylaxis of the Pfizer-BioNTech and the Moderna vaccines have been reported as 5.0 cases per million and 2.8 cases per million, respectively. ${ }^{27}$ Polyethylene glycol present in the lipid nanoparticle was suspected as one of the causes of drug allergy. ${ }^{28}$ However, more studies are required to identify the underlying mechanism. Recently, Greinacher et al reported that vaccination with ChAdOx1 nCov-19 can lead to the development of vaccine-induced immune thrombotic thrombocytopenia (VITT) mediated by platelet-activating antibodies against PF4 in rare cases. ${ }^{29}$ Furthermore, herpes zoster or pityriasis rosea (PR) and PR-like eruptions, Guillain-Barré syndrome, and myocarditis following mRNA COVID-19 vaccination have been reported. ${ }^{26,30,31}$ There will be clarity on the side effects with an increase in the available data.

In this study, cluster 6 represented public management and included keywords, such as public health, global health, health policy and ethics. Public health measures such as physical distancing, facemasks, hygiene measures, isolating the sick and quarantining their contacts remain the key weapons to reduce transmission before herd immunity can be achieved in the population by mass vaccination. ${ }^{32-35}$ However, the same social control measures might be implemented differently in different countries. Studies have proved that timely improvement in the response level of the government may advance the inflection point of the pandemic curve, thereby ensuring time for further stockpiling and vaccination. ${ }^{35}$

Among the top 100 most cited publications, 31 papers which reported the results of clinical trials of different vaccines, such as the BNT162b1 and BNT162b2 mRNA vaccines, mRNA-1273 vaccine, ChAdOx1 nCoV-19 vaccine (AZD1222), and recombinant adenovirus (rAd)-based vaccine, yielded more than 100 citations. Like cluster 4, it focused on the safety and efficacy of the vaccines.

The citation historiography graph revealed the changes in the research themes. We could identify a few important studies. Several research topics were proposed simultaneously, confirming the boom in research during the pandemic.

\section{Conclusions}

The highest number of publications was from the USA, followed by England, China and Germany. The main partners of the USA were China, England and Canada. The University of Maryland and Ugur Sahin from the University Medical Center of the Johannes Gutenberg University were the most cited institution and author, respectively. The Vaccines featured the highest number of papers. The most cited journal was the New England Journal of Medicine. The COVID-19 vaccine research hotspots were as follows: attitudes towards vaccination, immunoinformatics analysis, clinical research, effectiveness and side effects and public management of vaccines. The timing diagram revealed that the research hotspots focused on the side effects of vaccines and public attitudes towards vaccination. This novel comprehensive bibliometric analysis can help researchers and non-researchers to rapidly identify the potential partners, landmark studies and research topics within their domains of interest. Through this study, we hope to provide more data to combat the COVID-19 pandemic.

\section{Ethical Approval}

No ethical approval is required for such studies, as patients are not involved. 


\section{Author Contributions}

All authors made a significant contribution to the work reported, whether that is in the conception, study design, execution, acquisition of data, analysis and interpretation, or in all these areas; took part in drafting, revising or critically reviewing the article; gave final approval of the version to be published; have agreed on the journal to which the article has been submitted; and agree to be accountable for all aspects of the work.

\section{Funding}

This research did not receive funding from any public or private organizations/institutions.

\section{Disclosure}

The authors do not have any conflicts of interest to declare.

\section{References}

1. World Health Organization. Coronavirus disease 2019 (COVID-19) situation report; 2020.

2. World health organization coronavirus disease (COVID-19) dashboard; 2021. Available from: https://who.sprinklr.com/. Accessed October 6, 2021.

3. Leng Z, Zhu R, Hou W, et al. Transplantation of ACE2 (-) mesenchymal stem cells improves the outcome of patients with COVID-19 Pneumonia. Aging Dis. 2020;11(2):216-228. doi:10.14336/ AD.2020.0228

4. Wang M, Cao R, Zhang L, et al. Remdesivir and chloroquine effectively inhibit the recently emerged novel coronavirus $(2019-\mathrm{nCoV})$ in vitro. Cell Res. 2020;30(3):269-271. doi:10.1038/s41422-020-0282-0

5. Gao J, Tian Z, Yang X. Breakthrough: chloroquine phosphate has shown apparent efficacy in treatment of COVID-19 associated pneumonia in clinical studies. Biosci Trends. 2020;14(1):72-73. doi:10.5582/bst.2020.01047

6. Stringhini S, Wisniak A, Piumatti G, et al. Seroprevalence of anti-SARS-CoV-2 IgG antibodies in Geneva, Switzerland (SEROCoV-POP): a population-based study. Lancet. 2020;396 (10247):313-319. doi:10.1016/S0140-6736(20)31304-0

7. Ellegaard O, Wallin JA. The bibliometric analysis of scholarly production: how great is the impact? Scientometrics. 2015;105 (3):1809-1831. doi:10.1007/s11192-015-1645-z

8. Polack FP, Thomas SJ, Kitchin N, et al. Safety and efficacy of the BNT162b2 mRNA Covid-19 vaccine. $N$ Engl J Med. 2020;383 (27):2603-2615. doi:10.1056/NEJMoa2034577

9. Folegatti PM, Ewer KJ, Aley PK, et al. Safety and immunogenicity of the ChAdOx1 nCoV-19 vaccine against SARS-CoV-2: a preliminary report of a Phase $1 / 2$, single-blind, randomised controlled trial. Lancet. 2020;396(10249):467-478. doi:10.1016/S01406736(20)31604-4

10. Voysey M, Clemens SAC, Madhi SA, et al. Safety and efficacy of the ChAdOx1 nCoV-19 vaccine (AZD1222) against SARS-CoV-2: an interim analysis of four randomised controlled trials in Brazil, South Africa, and the UK. Lancet. 2021;397(10269):99-111. doi:10.1016/S0140-6736(20)32661-1

11. Baden LR, El Sahly HM, Essink B, et al. Efficacy and safety of the mRNA-1273 SARS-CoV-2 vaccine. $N$ Engl J Med. 2021;384 (5):403-416. doi:10.1056/NEJMoa2035389
12. Hannah Ritchie EM, Rodés-Guirao L, Appel C, et al. Coronavirus Pandemic (COVID-19). Published online at OurWorldInData.org; 2020. Available from: https://ourworldindata.org/coronavirus. Accessed October 6, 2021. [Online Resource].

13. Radanliev P, De Roure D, Walton R. Data mining and analysis of scientific research data records on Covid-19 mortality, immunity, and vaccine development - In the first wave of the Covid-19 pandemic. Diabetes Metab Syndr. 2020;14(5):1121-1132. doi:10.1016/j. dsx.2020.06.063

14. Yu Y, Li Y, Zhang Z, et al. A bibliometric analysis using VOSviewer of publications on COVID-19. Ann Transl Med. 2020;8(13):816. doi:10.21037/atm-20-4235

15. Brown A, Horton R. A planetary health perspective on COVID-19: a call for papers. Lancet. 2020;395(10230):1099. doi:10.1016/S01406736(20)30742-X

16. World Health Organization. Top ten threats to global health in 2019; 2019. Available from: https://www.who.int/emergencies/ten-threatsto-global-health-in-2019. Accessed December 12, 2019.

17. Fisher KA, Bloomstone SJ, Walder J, et al. Attitudes toward a potential SARS-CoV-2 vaccine: a survey of U.S. adults. Ann Intern Med. 2020;173(12):964-973. doi:10.7326/M20-3569

18. Puri N, Coomes EA, Haghbayan H, et al. Social media and vaccine hesitancy: new updates for the era of COVID-19 and globalized infectious diseases. Hum Vaccin Immunother. 2020;16 (11):2586-2593. doi:10.1080/21645515.2020.1780846

19. Xu Y, Zhang R, Zhou Z, et al. Parental psychological distress and attitudes towards COVID-19 vaccination: a cross-sectional survey in Shenzhen, China. J Affect Disord. 2021;292:552-558. doi:10.1016/j. jad.2021.06.003

20. Wang Q, Yang L, Jin $H$, et al. Vaccination against COVID-19: a systematic review and meta-analysis of acceptability and its predictors. Prev Med. 2021;150:106694. doi:10.1016/j. ypmed.2021.106694

21. Li J, Wang P, Tracey KJ, et al. Possible inhibition of GM-CSF production by SARS-CoV-2 spike-based vaccines. Mol Med. 2021;27(1):49. doi:10.1186/s10020-021-00313-3

22. Dong R, Chu Z, Yu F, et al. Contriving multi-epitope subunit of vaccine for COVID-19: immunoinformatics approaches. Front Immunol. 2020;11:1784. doi:10.3389/fimmu.2020.01784

23. Jain R, Jain A, Verma SK. Prediction of epitope based peptides for vaccine development from complete proteome of novel corona virus (SARS-COV-2) using immunoinformatics. Int J Pept Res Ther. 2021;27:1-12. doi:10.1007/s10989-021-10205-Z

24. Amirlak L, Haddad R, Hardy JD. et al. Effectiveness of booster BCG vaccination in preventing Covid-19 infection. Hum Vaccin Immunother;2021. 1-3. doi:10.1080/21645515.2021.1956228

25. World Health Organization. COVID-19 vaccines. Available from: https:/www.who.int/emergencies/diseases/novelcoronavirus-2019/ covid-19-vaccines. Accessed August 20, 2021.

26. Eid E, Abdullah L, Kurban M, et al. Herpes zoster emergence following mRNA COVID-19 vaccine. J Med Virol. 2021;93 (9):5231-5232. doi:10.1002/jmv.27036

27. Risma KA, Edwards KM, Hummell DS, et al. Potential mechanisms of anaphylaxis to COVID-19 mRNA vaccines. J Allergy Clin Immunol. 2021;147(6):2075-2082.e2072. doi:10.1016/j. jaci.2021.04.002

28. Troelnikov A, Perkins G, Yuson C, et al. Basophil reactivity to BNT162b2 is mediated by PEGylated lipid nanoparticles in patients with PEG allergy. J Allergy Clin Immunol. 2021;148(1):91-95. doi:10.1016/j.jaci.2021.04.032

29. Greinacher A, Thiele T, Warkentin TE, et al. Thrombotic thrombocytopenia after ChAdOx1 nCov-19 vaccination. $N$ Engl J Med. 2021;384(22):2092-2101. doi:10.1056/NEJMoa2104840

30. Drago F, Ciccarese G, Rebora A, et al. Human herpesvirus-6, -7, and Epstein-Barr virus reactivation in pityriasis rosea during COVID-19. J Med Virol. 2021;93(4):1850-1851. doi:10.1002/jmv.26549 
31. Rosenblum HG, Hadler SC, Moulia D, et al. Use of COVID-19 vaccines after reports of adverse events among adult recipients of Janssen (Johnson \& Johnson) and mRNA COVID-19 Vaccines (Pfizer-BioNTech and Moderna): update from the Advisory Committee on Immunization Practices - United States, July 2021. MMWR Morb Mortal Wkly Rep. 2021;70(32):1094-1099. doi:10.15585/mmwr.mm7032e4

32. Moore S, Hill EM, Tildesley MJ, et al. Vaccination and non-pharmaceutical interventions for COVID-19: a mathematical modelling study. Lancet Infect Dis. 2021;21(6):793-802. doi:10.1016/S1473-3099(21)00143-2

33. Sandmann FG, Davies NG, Vassall A, et al. The potential health and economic value of SARS-CoV-2 vaccination alongside physical distancing in the UK: a transmission model-based future scenario analysis and economic evaluation. Lancet Infect Dis. 2021;21 (7):962-974. doi:10.1016/S1473-3099(21)00079-7
34. Prem K, Liu Y, Russell TW, et al. The effect of control strategies to reduce social mixing on outcomes of the COVID-19 epidemic in Wuhan, China: a modelling study. Lancet Public Health. 2020;5(5): e261-e270. doi:10.1016/S2468-2667(20)30073-6

35. Ma Y, Mishra SR, Han XK, et al. The relationship between time to a high COVID-19 response level and timing of peak daily incidence: an analysis of governments' stringency index from 148 countries. Infect Dis Poverty. 2021;10(1):96. doi:10.1186/s40249021-00880-x

\section{Publish your work in this journal}

Infection and Drug Resistance is an international, peer-reviewed openaccess journal that focuses on the optimal treatment of infection (bacterial, fungal and viral) and the development and institution of preventive strategies to minimize the development and spread of resistance. The journal is specifically concerned with the epidemiology of antibiotic resistance and the mechanisms of resistance development and diffusion in both hospitals and the community. The manuscript management system is completely online and includes a very quick and fair peerreview system, which is all easy to use. Visit http://www.dovepress.com/ testimonials.php to read real quotes from published authors. 